\title{
Epidemien und gesellschaftliches Leben
}

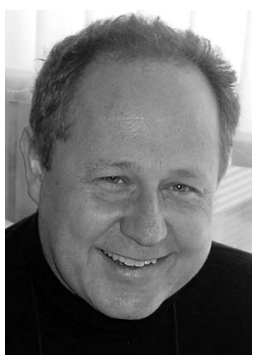

Manfred Wildner

\author{
Bibliografie \\ Gesundheitswesen 2021; 83: 5-7 \\ DOI 10.1055/a-1254-1249 \\ ISSN $\quad 0941-3790$
}

(C) 2021. Thieme. All rights reserved.

Georg Thieme Verlag KG, Rüdigerstraße 14,

70469 Stuttgart, Germany

\section{Korrespondenzadresse}

Prof. Dr. med. Manfred Wildner

Pettenkofer School of Public Health

c/o Bayerisches Landesamt für Gesundheit und Lebensmit-

telsicherheit

Veterinärstraße 2

85764 Oberschleißheim

Deutschland

Manfred.Wildner@Igl.bayern.de
Eine gern zitierte Bestimmung des Aristoteles beschreibt den Menschen als „geselliges Lebewesen“, ja geradezu als „politisches Tier“ (zoon politikon) [1]. In seiner Abhandlung über „Die politischen Dinge“ macht der altgriechische Philosoph das u. a. an der Fähigkeit des Menschen zur einsichtigen Rede fest, zur „Unterscheidung von Gut und Böse, Richtig und Falsch und zu anderen moralischen Qualitäten“. Diese gemeinsame Befähigung wiederum vereint die Menschen und befähigt sie so zu staatlicher Organisation - mehr noch: Damit ist gesellschaftliches Leben Teil der menschlichen Natur. Eine Einschätzung, die neben ihrer Anscheinsvalidität auch im Zentrum der modernen Politikwissenschaften und soziologischen Diskurstheorien steht und evolutionär bzw. soziobiologisch auch in der menschlichen Phylogenese verankert werden kann. Zu den o.g. „anderen Qualitäten“ gehören nach heutiger Erkenntnis insbesondere die sozialen Qualitäten Reziprozität und Empathie [2]. Damit darf der Mensch nicht nur als Individuum verstanden werden, sondern sollte ergänzend immer auch in seinen sozialen Bezügen und, da er mehr als nur ein zu sozialen Beziehungen befähigtes Tier ist, in seinen gesellschaftlichen Verhältnissen gesehen werden. Erst die menschliche Gesellschaft ermöglicht ein vollumfängliches menschliches Leben.

Wie ergibt sich hieraus ein Bezug zu Gesundheit? Zunächst könnte man an die bekannten Bestimmungen der Gesundheit als „biopsychosoziale“ Gesundheit denken. Aber ist unsere medizinische Versorgung nicht explizit auf das Individuum ausgelegt? Bilden das exklusive dyadische Vertrauensverhältnis von Arzt/Ärztin und Patient/in, der Respekt vor der Patientenautonomie und die zugehörige ärztliche Schweigepflicht nicht geradezu die medizinische Antithese zum sprechenden und sich munter sozialisierenden „zoon politikon“? Der sehr wohl gegebene Bezug wird augenfällig, wenn man einen Schritt zurücktritt und die speziellen individual- medizinischen Wissenschaftszweige wie Genomik, Epigenetik oder Zellularpathologie zugunsten eines größeren sozialen Kontextes übersteigt. Dies gilt nicht nur für die psychosozialen Bereiche von Psychiatrie, Psychotherapie und Psychosomatik, es gilt in einem wohlverstandenen sozialmedizinischen Verständnis für alle Erkrankungen [3]. Schon die Möglichkeit, sich ärztlich dem Individuum zuzuwenden, mit allen modernen Behandlungsverfahren, ist eine in jeder Hinsicht gesellschaftlich geschaffene Möglichkeit.

Um dies an der „epidemiologischen Triade“ beispielhaft für den Bereich der übertragbaren Krankheiten deutlich zu machen: Die Beschäftigung mit einer nur äußeren Ursache wie z. B. einem pathogenen Mikroorganismus in seinen mikrobiologischen Eigenschaften ist essentiell und von großem Wert, im Weiteren wird diese Ursache jedoch immer in Beziehung sowohl zu den durchaus unterschiedlichen menschlichen (Wirts-)Organismen und darüber hinaus auch zur zugehörigen natürlichen und sozialen bzw. zivilisatorischen Umwelt gesetzt werden müssen: Agent, Host, Environment - diese 3 bilden fortan den gedanklichen Rahmen. Und so mag eine Infektionskrankheit aus individualmedizinischer Betrachtungsweise zunächst biomedizinisch zu verstehen sein - Epidemien sind ein Gruppenphänomen und bei einer bevölkerungsmedizinischen Betrachtungsweise in erster Linie ein Phänomen gesellschaftlichen Lebens [4, 5]. Die Biomedizin ist im Übrigen selbst ebenfalls ein Phänomen dieses gesellschaftlichen Lebens: sie ist der Blick auf die biologische Natur mit den gesellschaftlich bereitgestellten Mitteln der Wissenschaft.

Epidemien als Phänomene gesellschaftlichen Lebens? Die griechische Wortbedeutung von Epidemie ist zunächst schnörkellos: „Das, was über (epi-) das Volk (demos) kommt“. Das, was in alten Zeiten über das Volk gekommen ist, dürften in erster Linie wohl tatsächlich übertragbare Krankheiten gewesen sein, auch wenn 
der Begriff heute auch für die nicht-übertragbaren Krankheiten verwendet wird.

Ein Grund, sich diesen Begriff „Epidemie“ näher anzusehen. Man könnte bei dem naheliegenden Ansatz beginnen, dass mit der Bezeichnung Epidemie zunächst eine Mehrzahl an aktuell oder potentiell Betroffenen gemeint ist. Ein solcher quantitativer Ansatz könnte dann eine überschaubare Häufung von Einzelerkrankungen im Rahmen eines umschriebenden Ausbruchs von einem größeren, dynamischen oder länger anhaltenden Geschehen in einer Region bzw. einer Bevölkerungsgruppe unterscheiden. Dieses wäre als Epidemie zu bezeichnen und könnte bisweilen sogar die Kriterien einer Pandemie erfüllen - einer „gesundheitlichen Notlage von internationaler Tragweite“. Letztere Feststellung würde allerdings von einer dazu legitimierten internationalen politischen Organisation getroffen - der Weltgesundheitsorganisation (WHO), einer 1948 insbesondere zur internationalen Seuchenbekämpfung gegründeten Sonderorganisation der Vereinten Nationen. Diese Feststellung müsste dann alle 3 Monate hinsichtlich ihres Fortbestandes überprüft werden.

Als gesundheitspolitisches bzw. administratives Werkzeug sind der WHO die Internationalen Gesundheitsvorschriften (International Health Regulations, IHR) an die Hand gegeben, welche Informationsweitergabe und Meldewesen, Vorbereitung auf Epidemien, präventive Maßnahmen, abgestimmtes Handeln während eines Ausbruchs und vieles andere mehr regeln. Die weltweite Eradikation der Pocken und die Elimination von Polio und Masern in großen Regionen sind nur einige der großen Erfolge dieser in ihrer Führungsleistung herausragenden gesundheitspolitischen Fachorganisation.

Beschränkt sich die Epidemie auf eine Region oder eine Bevölkerungsgruppe, in welcher sie dann aber anhaltend zu finden ist, wird sie als Endemie bezeichnet. Bezeichnungen ihrer Dynamik wiederum sind Explosivepidemie oder schleichend verlaufende Tardivepidemien, die auch als Mischepidemie zusammen auftreten können. Die Krankheitsfälle können sich um eine Punktquelle gruppieren oder als Streuepidemie weitverbreitet sein, z. B. bei einer Verbreitung über Lebensmittellogistikketten [7]. Diese Vielgesichtigkeit hat lange Zeit einer Vielzahl von Ursachenzuschreibungen Raum gegeben: Schlechte Luft (ital. mal'aria) und Zersetzungsprozesse im Boden mit sich daraus entwickelnden toxischen Miasmen waren 2 Jahrtausende, bis hin zu Max von Pettenkofers „Faktor X“, die führende Hypothese allerdings auch schon in Kenntnis der Weitergabemöglichkeit durch Menschen oder Waren.

Konsequent wurden daher auch soziale Absonderungsverfügungen gegenüber „unreinen“" Menschen über die Jahrtausende hinweg "politisch“ formuliert und teilweise in religiöse Gebote gegossen. In den mosaischen Gesetzen aus langer vorchristlicher Zeit finden sich solche Gebote, der „Schwarze Tod“ führte im 13. Jahrhundert zu mehrwöchigen Quarantänevorschriften bei einlaufenden Schiffen (ital. quaranta giorni - 40 Tage). Von daher besteht seit langem eine enge Verbindung zwischen Epidemien, Hygiene und gesellschaftlichen Ordnungslehren [8]. Erst das 19. Jahrhundert brachte - mithilfe neuer Technologien (Mikroskopie) und einer neuen wissenschaftlichen Vorgehensweise (Koch-Henlesche Postulate) - neue Klarheit. Zwar hatte 1854 Fillipo Pacini erstmals den Choleraerreger als mögliche Ursache dieser Erkrankung beschrie- ben. Die tatsächliche Kausalität wurde jedoch erst 30 Jahre später von Robert Koch mit Hilfe seiner kausalen Postulate als neuer wissenschaftlicher Methode überzeugend dargelegt. Loius Pasteur hatte schon 1865 mit seinem Mikroskop Parasiten als Ursache einer Erkrankung der Seidenraupe ausgemacht - die von ihm aufgestellte Keimtheorie erwies sich in Verbindung mit den Entdeckungen anderer Forscher zunehmend als universell gültiger Ansatz zum Verständnis und zur Kontrolle der Infektionskrankheiten. Die Infektionsschutzmaßnahmen gingen als technologische und politische Maßnahmen Hand in Hand mit einem verbesserten biomedizinischen Verständnis: Als Beispiel seien die politischen Beschlüsse zur (ab-)wassertechnischen Assanierung in Großstädten wie München, Hamburg oder London im 19. Jahrhundert genannt [9].

Der Choralvers „Media vita in morte sumus - Mitten im Leben sind wir im Tod" hatte seit alten Zeiten für alle Menschen gegolten. Der Nationen-übergreifende, inter-nationale wissenschaftliche Verständigungsprozess, basierend auf empirischer Beobachtung, ihrer kritischen Reflexion und einer ergebnisoffenen Diskussion entfaltete seit der Aufklärung zunehmend eine historisch bahnbrechende Wirkung. Er bescherte uns Menschen im 20. und 21. Jahrhundert die Erwartung eines langen Lebens - wohl erstmalig in der Menschheitsgeschichte. Das segensreiche Wirken naturwissenschaftlicher Forschung hält seither an, insbesondere auch in der Mikrobiologie und klinischen Infektiologie. Infektionsschutzmaßnahmen sind daher begleitet und geleitet von genomischen Analysen und darauf aufbauenden spezifischen molekularen Testmethoden, vom Wissen über hauptsächliche Infektionswege und ihrer physikalischen Beschreibung als Tröpfchen-, Aerosol- oder Schmierinfektion, bis hin zu innovativen Entwicklungen von Impfstoffen und Therapeutika u.v.a.m.

Und doch haben wir sie noch immer - Ausbrüche, Endemien, Epidemien, auch Pandemien, am Beginn des 21. Jahrhunderts. Und bei genauerem Hinsehen wird deutlich, dass der biomedizinische Ansatz, so segensreich er seit der Formulierung der Keimtheorie auch war, für einen nachhaltigen Infektionsschutz alleine nicht ausreicht. Was fehlt noch? Die Antwort wurde bereits formuliert: Man wird Epidemien und erst recht Pandemien erst dann gerecht werden, wenn man sie auch, gleichwertig zu einem naturwissenschaftlich-biologischen Verständnis, als Phänomene gesellschaftlichen Lebens aus einer systemischen Perspektive heraus begreift. Und was ist darüber hinaus in einer weiteren Perspektive nicht ebenfalls übertragbar: Sind Übergewicht, Bluthochdruck, Konsum illegaler Suchtmittel nicht auch übertragbar, in einem weiteren Sinn durch Vorlieben, sozial präferierte Lebensweisen, Angebote und Schwachstellen einer Gesellschaft [6]?

Eine solche weite Perspektive ist eine allgemeine Notwendigkeit für das Verständnis von Gesundheit und ihr folgen auch die Beiträge in diesem Heft, im Einzelnen und in ihrer Zusammenschau: Zur Bedeutung der „Big Five“-Persönlichkeitsmerkmale für die subjektive Gesundheit und Lebenszufriedenheit im Jugendalter und zur Entwicklung der selbstberichteten Gesundheit über die Sekundarstufe, zu Implementierungsmerkmalen psychiatrischer Modellvorhaben (nach §64b SGB V) in Schleswig-Holstein, zu Aufwand, Erfolg und Scheitern bei der Suche nach einem ambulanten Psychotherapieplatz, zur ambulanten Haus- und Facharztversorgung im ländlichen Raum in Deutschland, zur Offenheit der Bevölkerung 
für Teledermatologie in Bayern, zu regionalen und sozialen Disparitäten der Adipositas im zeitlichen Verlauf in Österreich und zum FIMA-Fragebogen zur Messung der Inanspruchnahme von Versorgungsleistungen anhand von Routinedaten der Krankenversicherung.

Um am Ende noch einmal zum Anfang zurückzukehren: Krankheit mag einen Einzelnen befallen, Epidemien betreffen immer Gruppen. Und so ist es höchste Zeit, die Aspekte der sozialen Public Health-Wissenschaften verstärkt in Prävention, Management und Nachsorge von Ausbrüchen und auch ganz allgemein verstärkt in das Verständnis von Epidemien einzubeziehen. Der gleiche Erreger befällt die Menschen einer politischen Gemeinschaft - das eine Mal wütet er in den Alten- und Pflegeheimen, das andere Mal haben sich kluge vorsorgliche soziale Schutzzonen um diese Unterkünfte gebildet. Menschliche Gesellschaften und staatliche Organisationen werden sich in der Qualität ihrer politischen Natur zu beweisen haben: Pandemien lassen sich nicht durch ungeeignete Massentestungen gleichsam ritualisiert „wegtesten “ [10], sie lassen sich nicht von populistischen Pressemeldungen beeindrucken und auch nicht durch Querdenken einfach wegdenken. Pandemien bringen unser politisches Vermögen oder Unvermögen auf den Punkt: Sie verlangen zu ihrer Bewältigung wissenschaftliche Klarheit und Wahrheit und eine daran anknüpfende verantwortungsvolle Politik jenseits demoskopisch inspirierter Rhetorik. Eben das, was uns als „zoon politikon“ im Kern ausmacht, verbindet und auch als Einzelwesen verpflichtet: Die schon angesprochene Fähigkeit des Menschen zur einsichtigen Rede, zur „Unterscheidung von Gut und Böse, Richtig und Falsch und zu anderen moralischen Qualitäten“.
Interessenkonflikt

Die Autorinnen/Autoren geben an, dass kein Interessenkonflikt besteht.

\section{Literatur}

[1] Aristotle, Politics, Book 1, section 1253a (tufts.edu). URL http://www. perseus.tufts.edu/hopper/text?doc $=$ Perseus:text:1999.01.0058:book $=1$ : section $=1253 \mathrm{a}$. accessed 02.12.2020

[2] De Waal F. Primaten und Philosophen. München: Carl Hanser; 2008

[3] Wildner M, Niehoff JU, Hoffmann W. Entwicklungslinien der Sozialmedizin und Öffentlichen Gesundheit in Deutschland. Gesundheitswesen 2016; 78: 113-119

[4] Delamater PL, Street E], Leslie TF et al. Complexity of the Basic Reproduction Number (R0). Emerging Infectious Diseases 2019; 25: $1-4$

[5] Niehoff JU. Epidemien übertragbarer Krankheiten. Berlin: SalusCon; 2020

[6] Kucharski A. The rules of contagion. London: Profile Books; 2020

[7] Robert Koch-Institut, Hrsg. RKI-Fachwörterbuch Infektionsschutz und Infektionsepidemiologie. Berlin: 2015

[8] Schipperges H. Gesundheit und Gesellschaft: Ein historisch-kritisches Panorama. Berlin: Springer; 2007

[9] Locher WG, von Pettenkofer Max. Sonderausgabe für die Pettenkofer School of Public health und die Landeshauptstadt München; 2018

[10] Andermann A, Blancquaert I, Beauchamp S et al. Revisiting Wilson and Jungner in the genomic age: a review of screening criteria over the past 40 years. Bull World Health Org 2008; 86: 317-319 